\title{
Interferon nomenclature
}

AT a recent, specially-convened meeting an international group of scientists attempted to devise a system to define and classify the interferons*. The following is the unanimous report from the group.

The committee accepted the definition for interferon: "To qualify as an interferon a factor must be a protein which exerts virus non-specific, antiviral activity at least in homologous cells through cellular metabolic processes involving synthesis of both RNA and protein."

The preferred abbreviation for inter feron is IFN, each interferon to be identified according to animal of origin: e.g. human (Hu IFN); murine (Mu IFN); bovine (Bov IFN); rat (Rat IFN); chicken, porcine, feline, equine interferon, etc. However, it may be necessary in some cases to apply generic nomenclature: e.g. monkey=Rhesus interferon, etc.; fish $=$ Salmo interferon, etc.; bat $=$ Taderida interferon etc.

Interferons will be classified into types on the basis of antigenic specificities, type designations to be alpha $(\alpha)$, beta $(\beta)$ and gamma $(\gamma)$, corresponding to previous designations of leukocyte (Le), fibroblas (F), and type II (immune) interferons, respectively. The old terminology of 'leukocyte', 'fibroblast' and 'immune' interferons were, by committee consensus, abolished, as they clearly were misnomers: both leukocyte and fibroblasts can produce each of these two types of interferon, and 'immune' interferons are often induced by mitogens rather than by immune recognition reactions. Also, the abbreviation ' $F$ ' has often been used to designate either fibroblast or fast migrating interferons. Alpha and beta interferons are usually acid-stable and correspond to what have

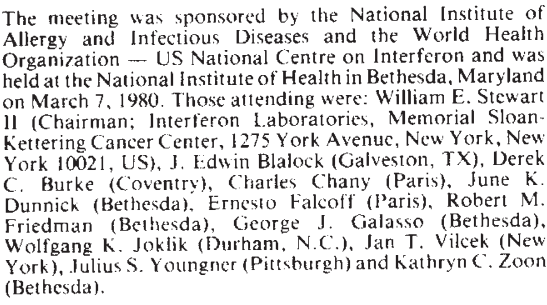

respiration is significant for marine phytoplankton (J. Burris, Pennsylvania State University). On a global average the fate of about $80 \%$ of the primary production is to be recycled within the photic zone. The relative importance in nutrient recycling of bacteria, microzooplankton, larger zooplankton and nekton in the photic zone appears to vary between the continental shelf, the slope water and the open ocean (W. G. Harrison, Bedford Institute, Dartmouth, Canada).

Harrison's paper is indicative of the been called type I IFNs; gamma interferons are usually acid-labile and correspond to what has been called type II IFNs. If there are discovered to be classes of IFN which are not presently recognized, they could be designated sequentially IFN-delta, epsilon, etc. The Table lists the new nomenclature for human and murine interferons and the corresponding designations which have been or are being employed. Interferon preparations may contain more than one type: for example, interferons derived from human lymphoblastoid cells and interferons from murine fibroblasts contain both IFN- $\alpha$ and IFN- $\beta$. These can be designated as to predominant type. Thus, the interferons presently employed in clinical trials are either HuIFN- $\alpha$ or HuIFN- $\beta$, or admixtures thereof.

The committee's recommendations apply at present only to human and murine IFNs, since there is insufficient information about IFNs from other animal species to allow differentiation into types. Workers using IFNs from other species are encouraged either to attempt preliminary classification based on antigenic homology with existing antisera specific for human or mouse alpha, beta or gamma IFNs, or to establish sequence homology with the known types of human and mouse interferons.

It is recognized that there may be size, charge, sequence and other heterogeneities within the designated IFN types. Currently, there is insufficient information available about the nature of these differences to allow designation of specific subtypes. Properly documented differences in molecular size appear to be useful parameters for differentiation until more stringent criteria (amino acid sequence, monoclonal antibodies) become available. Molecular weight designations may be indicated in parentheses, e.g. Hu IFN- $\alpha$ $(18 \mathrm{~K})$ or $\mathrm{Mu}$ IFN- $\beta(38 \mathrm{~K})$, or subtypes of IFNs based on specific amino acid sequence differences can be classified as $\mathrm{Hu}$ IFN- $\alpha_{1}$, HuIFN- $\beta_{2}$, etc.

Indication of interferon origin for those that behave antigenically similarly may also be helpful. Thus, interferon from human lymphoblastoid cells and interferon from human leukocytes are both classified antigenically as HuIFN- $\alpha$, yet these interferons differ in certain amino acids. Therefore, the nomenclature HuIFN- $\alpha$ (Ly) or HuIFN- $\alpha$ (Le) may be used until such time as specific differences in amino acid loci have been established and subtype designations can be assigned. Also, it is now recognized that there are a number of forms of HuIFN- $\alpha$ that differ in certain amino acids; eventually these may be designated according to specific amino acids and their locations. Schemes for such designation of subtypes of each of the major IFN types will be developed at subsequent meetings of the Nomenclature Committee. The next meeting of the committee will be held in April 1981. Suggestions and comments for consideration by the committee should be forwarded to the committee's Chairman.

The committee hopes that scientists and scientific journal editors will adopt the proposed nomenclature to avoid the proliferation of terms for the same substances and the indiscriminate naming of newly discovered factors.

Old and new nomenclature for human and mouse interferons

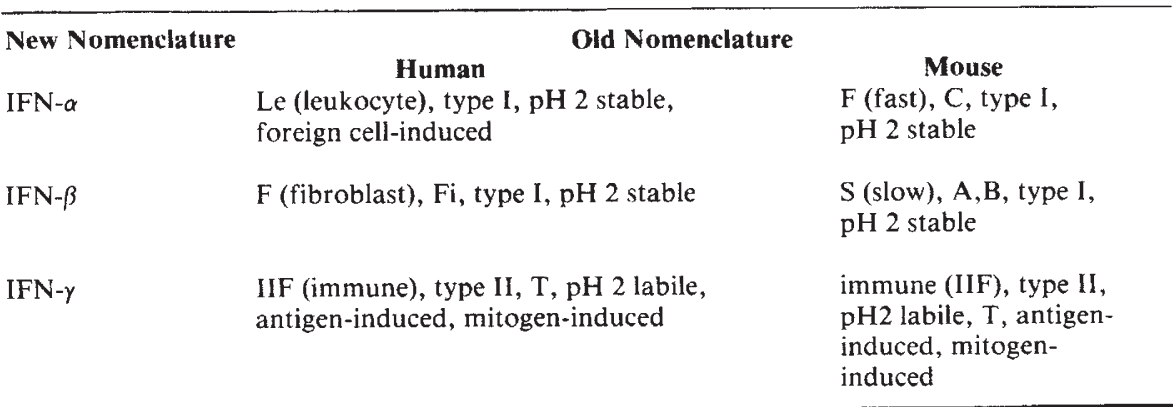

expanding interest of biological oceanographers in the fate of primary production. Globally, about $20 \%$ of it appears to sink out of the photic zone. J. Walsh (Brookhaven National Laboratory) postulated that a large fraction of this sinking flux on the continental shelf off New York is buried in the sediments of the upper continental slope, as shown by the relatively high sediment organic carbon content and a low $(\sim 6)$ ratio of carbon to nitrogen in the organic matter. Models of carbon flows in the circulation of the deep ocean $(M$.
Fiadeiro, Yale University, New Haven) suggest what the magnitude of the sinking flux of organic carbon may be. Unhappily, the experimental measurements of the flux in the North Pacific using particle interceptor traps are inconsistent with the models. Further collaboration among the several oceanographic disciplines seems required to clarify such problems and especially to define the significance of sinking organic particles as sinks and transporters of atmospheric carbon dioxide from fossil fuel burning. 